\title{
New advances in multiple autonomous aerial robots formation control technology
}

\author{
XU Yang ${ }^{1}$, LUO DeLin ${ }^{1 *}$, YOU YanCheng ${ }^{1} \&$ DUAN HaiBin ${ }^{2}$ \\ ${ }^{1}$ School of Aerospace Engineering, Xiamen University, Xiamen 361102, China; \\ ${ }^{2}$ School of Automation Science and Electrical Engineering, Beihang University, Beijing 100191, China
}

Received December 4, 2018; accepted January 21, 2019; published online July 18, 2019

\begin{tabular}{ll}
\hline Citation: & Xu Y, Luo D L, You Y C, et al. New advances in multiple autonomous aerial robots formation control technology. Sci China Tech Sci, 2019, 62: \\
1871-1872, https://doi.org/10.1007/s11431-018-9457-9
\end{tabular}

Autonomous aerial robotics has become a hot direction of research inside the community of robotics and control. The primary problem addressed by formation control is to steer multiple aerial robots to form desired geometric patterns and, at the same time, realize desired collective swarming behaviors in a decentralized or distributed manner. In contrast to ground vehicles, aerial robots have the ability to work in three-dimensional (3D) airspace. Equipped with electric or hydraulic motors, the vertical take-off and landing (VTOL) capability is a typical performance of aerial robots. Formation control technology for such aerial robots is incessantly springing up to satisfy the requirements of highly intelligent autonomous systems, which affects both military and civil areas, including missile defense, battlefield surveillance, satellite network construction, fire suppression, power grid inspection, commercial show, etc. [1-5]. Such a problem of multiple aerial robots formation control is exceptionally challenging to analyze if practical constraints such as complex dynamics, motion constraints, and imperfect measurements are incorporated.

The problem of formation control has been studied for several decades. The approaches proposed in the early stage such as behavior-based ones can handle complex formation tasks but are not able to guarantee system convergence. Ever since the successful application of the consensus theory in the formation control, tremendous research efforts have been devoted to developing convergence-guaranteed formation

*Corresponding author (email: luodelin1204@xmu.edu.cn) control approaches. Very recently, many new methods have been proposed to push the boundary of formation control technologies towards practical applications on aerial robots. This news aims to survey the most recent advances in the formation control, which can be directly or potentially applied to autonomous aerial robots.

Based on consensus theory as well as algebraic graph theory, innovative formation control schemes have been proposed in recent surveys [6,7]. Most of graphical formation control methods try to encode certain algebraic constraints related to the target configurations with specific Laplacian matrices distributedly, such as complex Laplacian, stress matrix, signed Laplacian, barycentric coordinate, and rigidity theories. Complex Laplacian-based formation control is confined in the plane due to the definition of the complex coordinate. Formation rotating maneuvers can be achieved by the barycentric coordinate-based approach, but the corresponding control law is quite complicated by introducing the coordinate transform matrix. Stress matrixbased and rigidity theories-based formation methods require the interaction networks to be undirected, and thus resultant strict graphical conditions are not suitable for implementations. Notably, by combing properties of both affine transformation and signed Laplacian, signed Laplacian-based formation control could be applied to any dimensional coordinate-free condition under generally directed interaction networks [8]. Furthermore, equipped with different onboard sensors, researchers can categorize the formation control into the position-, displacement-, bearing-, and distance-based 
methods. From the perspective of sensing abilities, positionbased formation control needs all agents to know the global coordinate information, which needs every agent inside the group to be complete all-round. Only a few of members are informed of global coordinate information with displacement-based formation control methods. Relative information among agents in respective local coordinates is demanded with bearing-based and distance-based formation protocols, but the design process of these approaches would be a particularly hard task due to the limited acquired information. Then, formation control approaches that considering practical constraints have also been introduced recently, such as nonholonomic motion constraints, obstacle, and inter-vehicle collision avoidance, velocity saturation constraints, and nonzero velocity constraints. Being constrained by imperfect measurements such as bearing, range, or angle measurements, formation control methods need to be modified by introducing various types of estimators or observers. Selecting only a part of a swarm to be manipulated by human pilots or intelligent planners is a hot topic of human-machine interaction technology and it is called the leader selection problem [9]. Besides, synchronization and hierarchical stability is another crucial control problem for a formation. By integrating superiorities of virtual structure and leader-follower structure, a two-layer formation-containment control may provide a reasonable solution when existing relatively complex interaction networks [10]. Furthermore, formation maneuver control can be used to achieve various collective behaviors such as translational, rotational, and scaling motions in arbitrary dimensions. Therefore, both constant and time-varying formation maneuvers can be adjusted to complete complex tasks in obstacle-strewn environments.

To integrate the formation control into aerial robotic systems, here we provide the hierarchical structure of design flow in most of the recent existing literatures. In Figure 1, we demonstrate a typical hierarchical structure of $N$ aerial ro-

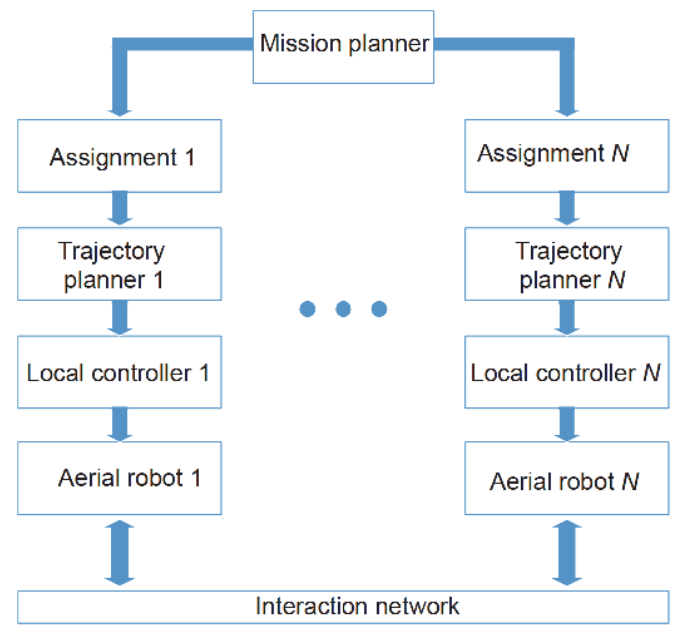

Figure 1 (Color online) Hierarchical structure of $N$ aerial robotic formation systems. botic formation systems. The first step is the concurrent mission planning with a distributed assignment. For instance, in a homogeneous formation, an optimal terminal constraint for formation reconfiguration control needs a simultaneous optimal assignment. Then the step for trajectory generation and motion planning is followed. In this step, collision avoidance and collision-free motions are considered to maximize the performance of the formation, like preventing the increase of the time to complete the arranged assignment. In the final step, distributed local formation controllers, sometimes called formation tracking controllers or formation maneuver tracking controllers, are designed to drive every member in aerial robotic systems to follow the prescribed trajectories. As aerial robotic formations are deployed to a greater extension for aggressive tasks, the design flow will be gradually updated bottom up from the subsystem level to the system level, and finally to the swarm level.

In this news, we survey the recent development in the formation control technology for autonomous aerial robots. Also, from the hierarchical division such that the formation control technology is the bottom level of aerial robotic systems, the convergence and stability are critical to the mission completeness. Many problems of formation control technology are still open, including heterogeneous aerial/ground robots cooperation, inter-swarm conflict, and counter-swarm technique. We expect that this news provides a useful overview for anyone who will carry out researches in aerial robotic systems.

This work was supported by the National Natural Science Foundation of China (Grant Nos. 61673327, 51606161, 11602209, 91441128), and the Natural Science Foundation of Fujian Province, China (Grant No. 2016J06011).

1 Xing J J, Tang G J, Cheng W K, et al. Robust nonlinear control of spacecraft formation flying using constraint forces. Sci China Tech Sci, 2011, 54: 2276-2282

2 Duan H B, Luo Q N, Yu Y X. Trophallaxis network control approach to formation flight of multiple unmanned aerial vehicles. Sci China Tech Sci, 2013, 56: 1066-1074

$3 \mathrm{Wu} \mathrm{Y,} \mathrm{Zhu} \mathrm{F,} \mathrm{Song} \mathrm{C.} \mathrm{Optimal} \mathrm{discretization} \mathrm{of} \mathrm{feedback} \mathrm{control} \mathrm{in}$ missile formation. Aerospace Sci Tech, 2017, 67: 456-472

4 Dong X, Zhou Y, Ren Z, et al. Time-varying formation tracking for second-order multi-agent systems subjected to switching topologies with application to quadrotor formation flying. IEEE Trans Ind Electron, 2017, 64: 5014-5024

$5 \mathrm{Xu} \mathrm{Y,} \mathrm{Lai} \mathrm{S,} \mathrm{Li} \mathrm{J,} \mathrm{et} \mathrm{al.} \mathrm{Concurrent} \mathrm{optimal} \mathrm{trajectory} \mathrm{planning} \mathrm{for}$ indoor quadrotor formation switching. J Intell Robot Syst, 2019, 94: 503-520

6 Oh K K, Park M C, Ahn H S. A survey of multi-agent formation control. Automatica, 2015, 53: 424-440

7 Zhu B, Xie L, Han D, et al. A survey on recent progress in control of swarm systems. Sci China Inf Sci, 2017, 60: 070201

8 Lin Z, Wang L, Chen Z, et al. Necessary and sufficient graphical conditions for affine formation control. IEEE Trans Automat Contr, 2016, 61: 2877-2891

9 Zhao S. Affine formation maneuver control of multiagent systems. IEEE Trans Automat Contr, 2018, 63: 4140-4155

10 Li D, Zhang W, He W, et al. Two-layer distributed formation-containment control of multiple Euler-Lagrange systems by output feedback. IEEE Trans Cybern, 2019, 49: 675-687 